\title{
Human Outer Solar System Exploration via $Q$-Thruster Technology
}

\author{
B. Kent Joosten \\ Associate, MBO Partners, Inc. \\ 2383 York Harbour Ct. \\ League City, TX 77573 \\ 281-543-7043 \\ kent.joosten@icloud.com
}

\author{
Harold G. "Sonny" White \\ NASA Johnson Space Center \\ 2101 NASA Parkway \\ Houston, TX 77058 \\ 281-483-0178 \\ harold.white-1@nasa.gov
}

\begin{abstract}
Propulsion technology development efforts at the NASA Johnson Space Center continue to advance the understanding of the quantum vacuum plasma thruster (QThruster), a form of electric propulsion. Through the use of electric and magnetic fields, a Q-thruster pushes quantum particles (electrons/positrons) in one direction, while the $Q$ thruster recoils to conserve momentum. This principle is similar to how a submarine uses its propeller to push water in one direction, while the submarine recoils to conserve momentum. Based on laboratory results, it appears that continuous specific thrust levels of $0.4-4.0 \mathrm{~N} / \mathrm{kWe}$ are achievable with essentially no onboard propellant consumption.
\end{abstract}

To evaluate the potential of this technology, a mission analysis tool was developed utilizing the Generalized Reduced Gradient non-linear parameter optimization engine contained in the Microsoft Excel ${ }^{\circledR}$ platform. This tool allowed very rapid assessments of "Q-Ship" minimum time transfers from earth to the outer planets and back utilizing parametric variations in thrust acceleration while enforcing constraints on planetary phase angles and minimum heliocentric distances. A conservative Q-Thruster specific thrust assumption (0.4 N/kWe) combined with "moderate" levels of space nuclear power (1 - 2 MWe) and vehicle specific mass $(45-55 \mathrm{~kg} / \mathrm{kWe})$ results in continuous milli-g thrust acceleration, opening up realms of human spaceflight performance completely unattainable by any current systems or near-term proposed technologies. Minimum flight times to Mars are predicted to be as low as 75 days, but perhaps more importantly new "retro-phase" and "gravity-augmented" trajectory shaping techniques were revealed which overcome adverse planetary phasing and allow virtually unrestricted departure and return opportunities. Even more impressively, the Jovian and Saturnian systems would be opened up to human exploration with round-trip times of 21 and 32 months respectively including 6 to 12 months of exploration at the destinations. Finally, interstellar trip times are assessed at milli-g acceleration levels.

\section{TABLE OF Contents}

1. INTRODUCTION ..................................................1

2. Q-THRUSTER SYSTEM BEHAVIOR ...................2

3. MARS MisSIONS................................................4

4. EXTREME PeRformanCE MisSiOnS................7

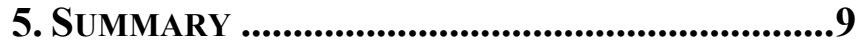

REFERENCES................................................99

BIOGRAPHY ....................................................9

APPENDIX A ........................................................11

APPENDIX B .......................................................12

APPENDIX C ..............................................13

\section{INTRODUCTION}

\section{Q-Thruster Overview}

It is not the intent here to detail the theory or engineering of quantum vacuum plasma thrusters (Q-Thrusters). Rather, an overview of the foundational physics and laboratory findings are given.

Q-Thrusters attempt to use the properties of the "quantum vacuum" to propel a spacecraft. Quantum Electrodynamics (QED) predicts that the quantum vacuum (the lowest state of the electromagnetic field) is not empty, but rather a sea of virtual particles and photons that pop into and out of existence stemming from the Heisenberg uncertainty principle. A number of approaches to utilize this quantum vacuum to transfer momentum from a spacecraft to the vacuum have been synopsized in [1].

A Q-Thruster uses the same principles as conventional plasma thrusters, namely magnetohydrodynamics, where plasma is exposed to crossed electric and magnetic fields which induce a drift of the entire plasma in a direction orthogonal to the applied fields. The difference arises in that a Q-Thruster uses quantum vacuum fluctuations as the "propellant" source, eliminating the need for conventional on-board propellant. A discussion of spacecraft "conservation of energy" is given in Appendix A. Recent laboratory test results [2] indicate the expected thrust-to-power ratio for flight applications could be in the $0.4-4.0 \mathrm{~N} / \mathrm{kWe}$ range, which is one to two orders of magnitude greater than current operational electric thrusters. This combination of characteristics - relatively high specific thrust combined with essentially zero on-board propellant requirement suggest space mission performance levels significantly exceeding current capabilities.

\section{Mission Analysis Approach}

The following analyses are intended to investigate the potential of Q-Thruster performance in the context of human exploration of the outer solar system. A parametric understanding of the interplay of thruster, vehicle and mission characteristics is first developed followed by specific instantiations of round-trip missions to Mars, Jupiter and Saturn along with investigations of several unique mission capabilities. Finally, a brief discussion of interstellar mission performance is offered. 


\section{Q-THRUSTER SYSTEM BEHAVIOR}

To evaluate the potential of Q-Thrusters in the human exploration of the outer solar system, an understanding of generalized system behavior is in order. The unique QThruster characteristics described above imply a nearly constant-mass spacecraft with thrust level varying linearly with thruster power input. While required power levels and system specific masses are some of the desired findings from the following analyses, it is clear that for outer planet missions, especially those beyond Mars, reasonable power levels can only be maintained with nuclear systems. Since the output power of such systems can be held constant (independent of heliocentric distance) the spacecraft will experience essentially constant thrust acceleration.

Excellent continuous-thrust mission analysis tools exist, however many of these require specialized operator expertise and exhibit levels of fidelity unnecessary for rapid assessment of a broad range of mission and vehicle parameters. For this high-level analysis, a closed-form analytical solution exists for the minimum time, phase independent orbit-to-orbit heliocentric transfer of a constant thrustacceleration spacecraft. To evaluate more complex missions with additional constraints, a computational tool was constructed utilizing the programmability and optimization capabilities of Microsoft Excel ${ }^{\circledR}$. Results from this analytical solution and numerical tool were compared with classic test cases and with a sophisticated NASA production trajectory optimizer.

\section{Heliocentric Analytical Solution}

Prior to the availability of computationally rapid numerical trajectory integration and calculus of variation analysis tools, various techniques were investigated to analytically approximate optimal continuous thrust trajectories [3], [4]. One somewhat successful method [5] sought to identify characteristic distances or velocity changes which, when used in rectilinear motion equations satisfying the specific problem's optimality conditions (usually minimum propellant usage with specified terminal position and velocity constraints) gave reasonable approximations to the propellant usage and flight times obtained from the more rigorous tools. These analytical solutions could become quite cumbersome however, since traditional propulsion systems provide varying acceleration levels due to propellant depletion and the propellant-optimal solutions for such acceleration profiles are thrust-coast-thrust arcs.

In our analysis however, the constant thrust acceleration condition provides exceedingly simple closed-form solutions to the problem of a spacecraft transferring from earth's heliocentric orbit to that of an outer planet without phase angle constraints. If one assumes circular, coplanar planetary orbits, the minimum acceleration levels corresponding to a given flight time are

$$
\begin{gathered}
a_{r}=\frac{4\left(r_{\text {target }}-r_{\text {earth }}\right)}{t_{f}^{2}}, \\
a_{t}=\frac{\sqrt{\frac{\mu_{\odot}}{r_{\text {earth }}}}-\sqrt{\frac{\mu_{\odot}}{r_{\text {target }}}}}{t_{f}}, \\
a_{\text {thrust }}^{2}=a_{r}^{2}+a_{t}^{2} .
\end{gathered}
$$

Here, $r_{\text {earth }}$ and $r_{\text {target }}$ are the heliocentric orbital radii of the earth and the target planets respectively, and $\mu_{\odot}$ is the sun's gravitational parameter. The $a_{r}$ term is immediately recognizable as the "field-free" constant acceleration magnitude required to traverse the radial distance between the planets' orbits in time $t_{f}$ assuming zero radial velocity at each end and a reversal of acceleration direction at the midpoint. Similarly, $a_{t}$ is simply the field-free constant tangential acceleration needed to match the target planet's orbital velocity over the same time interval. There is no tangential position constraint since this problem was defined as phase independent. It is a fairly trivial exercise to prove that these rectilinear solutions satisfy the optimality conditions and terminal state constraints. Their validity to the "true" dynamics is based on the observation that $a_{t}$ maintains the spacecraft in a gravitational "pseudo-equilibrium" such that radial gravitational accelerations can be ignored. Even so, upon evaluation $a_{t}$ contributes less that $1 \%$ to the magnitude of $a_{\text {thrust }}$ for all outer planet missions and thrust acceleration levels of interest, so can therefore be neglected. This allows the minimum flight time for a given constant thrust acceleration level to be expressed as

$$
t_{\text {min }}=2 \sqrt{\frac{r_{\text {target }}-r_{\text {earth }}}{a_{\text {thrust }}}} .
$$

The validity of the analytic solution will be demonstrated subsequently.

\section{Heliocentric Vehicle and Mission Parameterization}

For a Q-Thruster propelled nuclear-powered spacecraft the sole parameter affecting the vehicle dynamics is the (constant) thrust acceleration level and the associated steering policy. The thrust level is determined by the Q-Thruster specific thrust $T_{s}(\mathrm{~N} / \mathrm{kWe})$ and the power $P$ supplied to it. The spacecraft mass can be segregated into that associated with the propulsion and power systems and that associated with the payload $m_{p / l}$. Typically, for nuclear electric spacecraft parametric analysis, the propulsion and power systems are characterized by specific mass $\alpha(\mathrm{kg} / \mathrm{kWe})$. This parameter does tend to change with power level (due to economies of scale), but for modest power variations can be considered constant. Therefore, the vehicle thrust acceleration level can be expressed by 


$$
a_{\text {thrust }}=\frac{T}{m}=\frac{T_{s}}{m_{p / l} / P+\alpha} .
$$

This expression combined with that for $t_{\min }$ above gives a very quick way to estimate minimum heliocentric transit times given destination, Q-Thruster specific thrust, vehicle specific mass, power level and payload mass.

\section{Heliocentric Numerical Analysis}

To more rigorously examine aspects of Q-Thruster performance, an analysis technique was needed that still allowed relatively rapid assessments of vehicle and mission variations, but avoided the unnecessary complexity of production-level tools. Constant acceleration heliocentric twodegree-of-freedom vehicle equations of motion (Appendix B) were programmed into Microsoft Excel ${ }^{\circledR}$ and combined with the built-in General Reduced Gradient (GRG) parameter optimizer [6]. Several test cases were run including a classic low-thrust optimal control problem [7], [8], the solution to which the GRG/Excel ${ }^{\circledR}$ tool duplicated to a high degree of precision (Appendix C).

The first task was to verify the behavior predicted by the analytical solutions. GRG allows terminal constraints to be imposed on the state variables - in this case the final heliocentric radius along with final radial and tangential velocities. The parameters to be optimized were the thrust direction at the integration nodes and the duration of the trajectory. The trajectory duration was also the objective function to be minimized. Thrust acceleration magnitudes were selected to span the same range examined by the analytical assessment for transfers to Mars, Jupiter and Saturn.

Secondly, representative transfers to the three planets were assessed using NASA's "Copernicus" trajectory optimizer [9]. Copernicus is an extremely sophisticated six degree-offreedom tool that accurately models planetary ephemerides and multi-body gravity effects. It is capable of utilizing impulsive, finite and continuous thrust control variables, evaluating multiple performance indices and enforcing terminal state constraints.

\section{Comparison of Analysis Techniques}

Figure 1 compares results for minimum time, phase independent orbit-to-orbit heliocentric transfers of a constant thrust-acceleration spacecraft from the analytical, GRG/Excel ${ }^{\circledR}$ and Copernicus analyses, with acceleration expressed in milli-g's. As can be seen, there is excellent agreement amongst all techniques with less than 3\% minimum transit time difference between GRG/Excel ${ }^{\circledR}$ and Copernicus and $5 \%$ between the analytic solution and Copernicus. This comparison is also combined nomographically with the vehicle and mission parametrics described in Equation (5), allowing a visual interpretation of the sensitivity of flight time to the various quantities. The values of QThruster specific thrust span the range consistent with laboratory results.

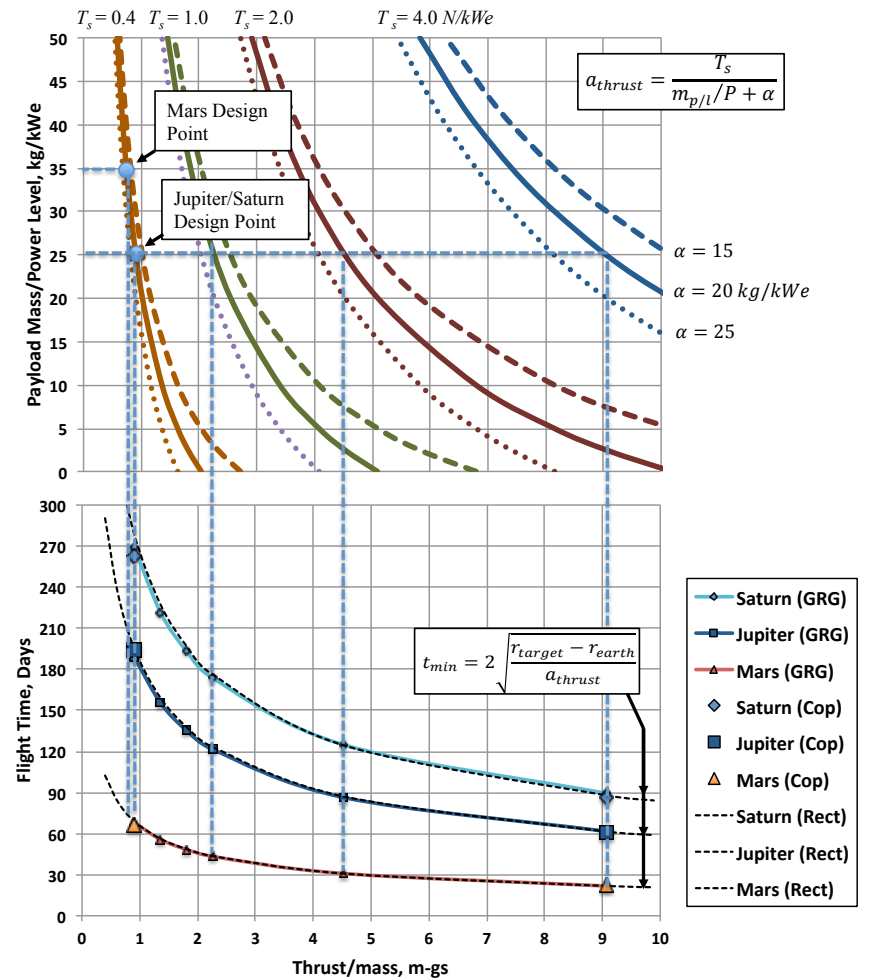

Figure 1 - Q-Ship Phase Independent Performance

It is apparent that there is a "knee of the curve" in minimum flight time vs. thrust acceleration at around 0.5-1.5 milli-g's which is achievable even at the lower range of the expected Q-Thruster specific thrust. At lower acceleration levels, flight times increase sharply, while at higher levels they decrease, but somewhat asymptotically. This performance region is therefore of interest in defining desirable thruster, vehicle and mission capabilities. Also, from the nomograph it is interesting to note that all other things being equal, simply increasing power level (decreasing $m_{p / l} / P$ ) does not strongly decrease flight time because while doing so would increase thrust, it would also increase vehicle mass due to larger power and thruster systems. However, it should be recognized that there is likely a tendency for $\alpha$ to decrease if power levels increase significantly [10].

\section{Planetocentric Phase}

A common technique to approximate the propellant consumption of low-thrust spacecraft during the multirevolution spiral from planetary orbit to escape velocity is the so-called Edelbaum transfer [11]. The equation of interest describes the equivalent ideal "field-free" velocity change $\Delta v_{\text {ideal }}$ experienced by a spacecraft transiting from a circular orbit of radius $r_{1}$ to another circular orbit of radius $r_{2}$ with a differential orbital inclination of $\Delta i$ :

$$
\Delta v_{\text {ideal }}^{2}=\frac{\mu}{r_{1}}+\frac{\mu}{r_{2}}-\frac{\mu}{\sqrt{r_{1} r_{2}}} \cos \frac{\pi}{2} \Delta i .
$$

Here, $\mu$ is the planet's gravitational parameter. For the case of departure from $r_{1}$ to escape velocity, $r_{2}=\infty$. Typically, 
$\Delta v_{\text {ideal }}$ is used to estimate the spacecraft propellant requirements via the "rocket equation," but since Q-Thruster spacecraft consume no propellant, we will use $\Delta v_{\text {ideal }}$ to estimate the flight time to achieve escape velocity $\Delta t_{e s c}$ through

$$
\Delta t_{\text {esc }}=\frac{\Delta v_{\text {ideal }}}{a_{\text {thrust }}}=\frac{\sqrt{\mu / r_{1}}}{a_{\text {thrust }}} .
$$

Since $a_{\text {thrust }}$ is constant for Q-Thruster spacecraft, Equation (7) is equally valid for planetary orbit departure or arrival. Examples of $\Delta t_{e s c}$ vs. $a_{\text {thrust }}$ are shown in Figure 2 for the following planetary orbits of interest: Earth: $400 \mathrm{~km}$ circular, Mars: $400 \mathrm{~km}$ circular, Jupiter: 1,882,700 km circular (Callisto) and Saturn: 273,948 and 1,221,870 km circular (Enceladus and Titan, respectively).

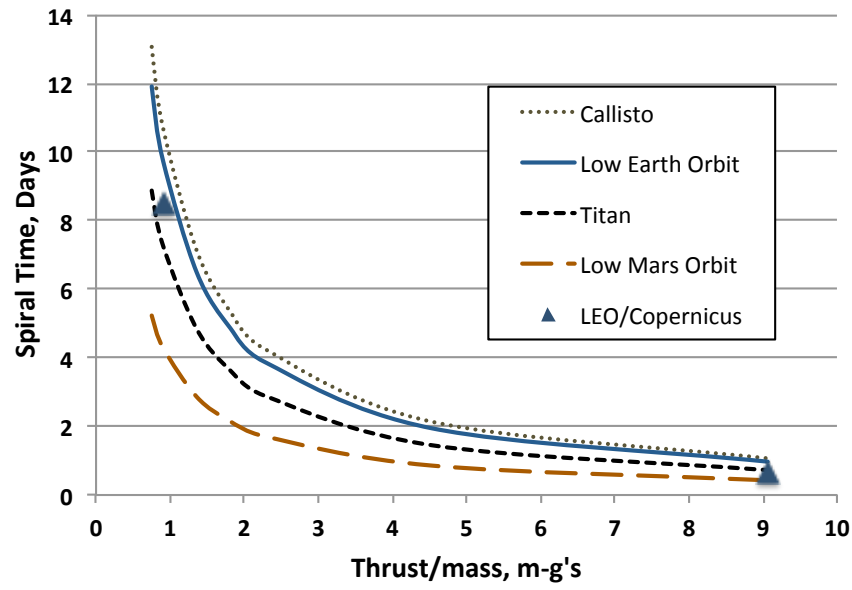

Figure 2 - Edelbaum Departure/Capture Spiral Times

In addition, two Copernicus assessments of Earth departure are shown. These minimum-time trajectories reach escape velocity $15-30 \%$ faster than the Edelbaum transfers predict. Upon examining the Copernicus profiles it was clear that at the acceleration levels chosen, the optimal steering profiles exhibited considerable radial thrust components, and since one of the tenets of the Edelbaum approximation is tangential steering, these results are not surprising. However, for preliminary mission assessments even at thrust levels of $\sim 1$ milli-g or higher, the Edelbaum computations are deemed acceptable, if conservative.

As can be seen from Figure 2 these data also display a "knee of the curve" at around 1 milli-g. This is an encouraging finding - if a spacecraft could achieve this thrust acceleration level, it would attain the more "asymptotic" mission times associated with both planetary departure/arrival and heliocentric transfers.

Now that the system behavior of Q-Thruster-based missions to the outer planets has been explored, the validity of the GRG/Excel ${ }^{\circledR}$ tool verified and the utility of the Edelbaum transfer demonstrated, subsequent analysis will focus on specific examples of vehicles and missions in order to more explicitly understand the performance afforded by this technology.

\section{MARS Missions}

\section{Vehicle and Mission Parameters}

Returning to Figure 1, we examine combinations of system and mission parameters that could allow nearly asymptotic performance levels and compare these against those considered state-of-the-art or which represent reasonable technological extrapolations. Previous NASA crewed Mars mission analyses [12] have provided estimates for the masses of the spacecraft systems associated with crew habitation assuming fairly high efficiency levels of water and air recovery. Parametric sizing tools indicate a habitation mass of 35 metric tons should be able to sustain 6-8 crewmembers for one year. The remainder of the spacecraft mass (power and propulsion systems) is derived from additional mass estimates [13]. The power system specific mass chosen corresponds to "Growth SP-100" technology (single lithium-cooled reactor with single-phase Brayton power conversion). The Q-Thruster-based propulsion system was assumed to exhibit a specific mass similar to that of ion thrusters of comparable power level. The aggregate power/propulsion $\alpha$ selected was $20 \mathrm{~kg} / \mathrm{kWe}$. As previously discussed, laboratory results indicate Q-Thruster specific thrust values could range from 0.4 to $4.0 \mathrm{~N} / \mathrm{kWe}$. In this analysis we use the more conservative value of 0.4 $\mathrm{N} / \mathrm{kWe}$.

Utilizing the above parameter choices and Equation (5) we can compute the power level necessary to achieve $a_{\text {thrust }}$ near the knee of the curve in Figure 1. Utilizing $1 \mathrm{MWe}$ of power results in $a_{\text {thrust }}=0.74$ milli-g's and a total vehicle mass of 55 tons. This is the value selected for the Mars mission assessments.

\section{Phase-Independent Transits}

It is immediately obvious from Figure 1 that this vehicle and payload combination gives a very impressive heliocentric phase-independent transit time to Mars of only 75 days. The trajectory and thrust profile computed from the GRG/Excel ${ }^{\circledR}$ tool are shown in Figure 3. Note that the optimal thrust profile is very similar to the radial "fieldfree" analytical solution from Equation (1) - nearly constant radial outward thrusting with a rapid switch to inward at the transit's halfway point. The arrows in the trajectory diagram indicate the direction of the thrust vector.

Also, by examining Equation (7) or Figure 2 it can be seen that only 12 days would be utilized spiraling up from a 400 km low Earth orbit to achieve escape velocity and only 5 days spiraling down to a $400 \mathrm{~km}$ low Mars orbit. These relatively rapid transits would argue for mission strategies where the "Q-Ship" operates between the lowest orbits possible to minimize the launch requirements of crew and supplies from earth and lander complexity at Mars. It also obviates the need for a high-speed entry vehicle to be hauled round-trip just to return the crew to the Earth. By quickly spiraling into Earth orbit at the end of the mission, the crew could readily be retrieved via a "ground-up" launch. 

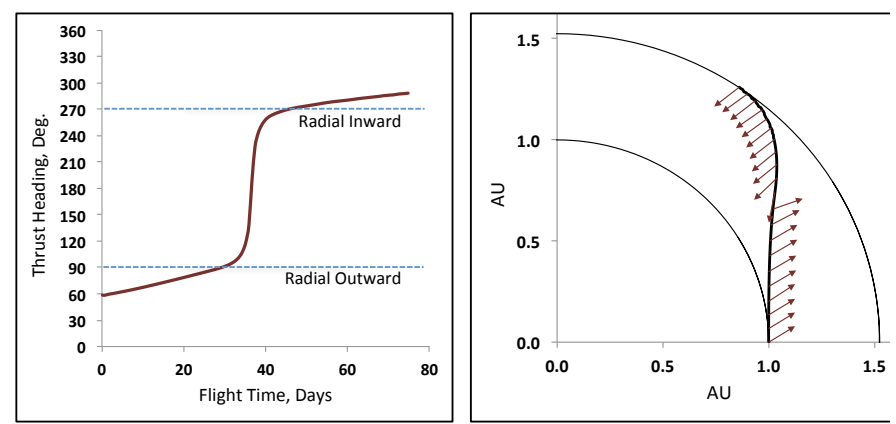

Figure 3 - Optimal Phase-Independent Earth-Mars Transfer, $T_{S}=0.4, P=1.0 \mathrm{MWe}, m_{p / l}=35 \mathrm{t}, \alpha=20 \mathrm{~kg} / \mathrm{kWe}$

While minimum transit time is an important metric in the evaluation of the potential of Q-Thrusters, it may not be the only or even the most important. Other advanced propulsion technologies [14] have focused on minimum transit time, but the challenges associated with the accompanying system or mission parameters (required power level, specific masses, mission risk) required to achieve those transit times may outweigh the potential propulsion system benefits.

In particular, focusing on minimum transit time, either outbound or inbound, usually implies optimal planetary alignment (phase angle) which occurs only once per Earthtarget synodic cycle - for Earth-Mars this is approximately 26 months. This low mission frequency seriously underutilizes a system with the extreme performance capability and multi-mission potential of the Q-Ship. The paradigm of a vehicle that transports a crew to Mars, loiters in orbit for months or years waiting for the correct planetary alignment and then transports the crew home needs to be rethought.

\section{"Any-Phase" Transits}

Mission analyses involving Mars vehicle concepts utilizing conventional or even advanced propulsion assumptions rarely examine departure or return opportunities that exceed the optimum planetary phasing by more than a few weeks. This is due to the extreme propellant penalties incurred by the associated velocity change increases. The "propellantless" Q-Thrusters would not suffer from this exponential mass penalty, but instead experience increasing transit times. We now examine the consequence of "any-phase" departure (and by symmetry, return) opportunities to extend the utility of Q-Thruster transportation.

The optimum phase-independent transfer shown in Figure 3 resulted in a departure Earth-Mars phase angle (Mars "leading" Earth is positive) of $15^{\circ}$. As we constrain this angle to be more positive (corresponding to an earlier departure date) the Q-Ship will need to extend its transfer time to compensate. As the phase angle is constrained to be more and more positive, the trajectory will begin to descend below $1 \mathrm{AU}$ in order to achieve higher angular rates through orbital dynamics effects.

Eventually, solar thermal conditions will limit this method of phase compensation. While the value of this minimum solar distance will ultimately depend upon vehicle design details, we can observe the effects of a constrained heliocentric radius on the optimal flight times. Fortunately, GRG allows inequality constraints to be imposed on state variables, and an arbitrary minimum heliocentric radius of 0.72 AU (Venus' orbit) was imposed. When adverse phasing becomes sufficiently severe, the vehicle transitions to what we have termed "gravity-augmented phasing", where all of the vehicle's thrust is directed radially inward, augmenting the sun's gravity and allowing faster heliocentric angular motion along the constrained radius than purely ballistic motion would allow. In the current example, our Q-Ship would be travelling in Venus' heliocentric orbit, but nearly $30 \%$ faster than the planet. This technique could be sustained indefinitely, eventually overcoming any degree of adverse Earth-Mars phasing at the expense of extended flight time. Figure 4 depicts gravity-augmented phasing as the vehicle compensates for an initial phase angle of $180^{\circ}$

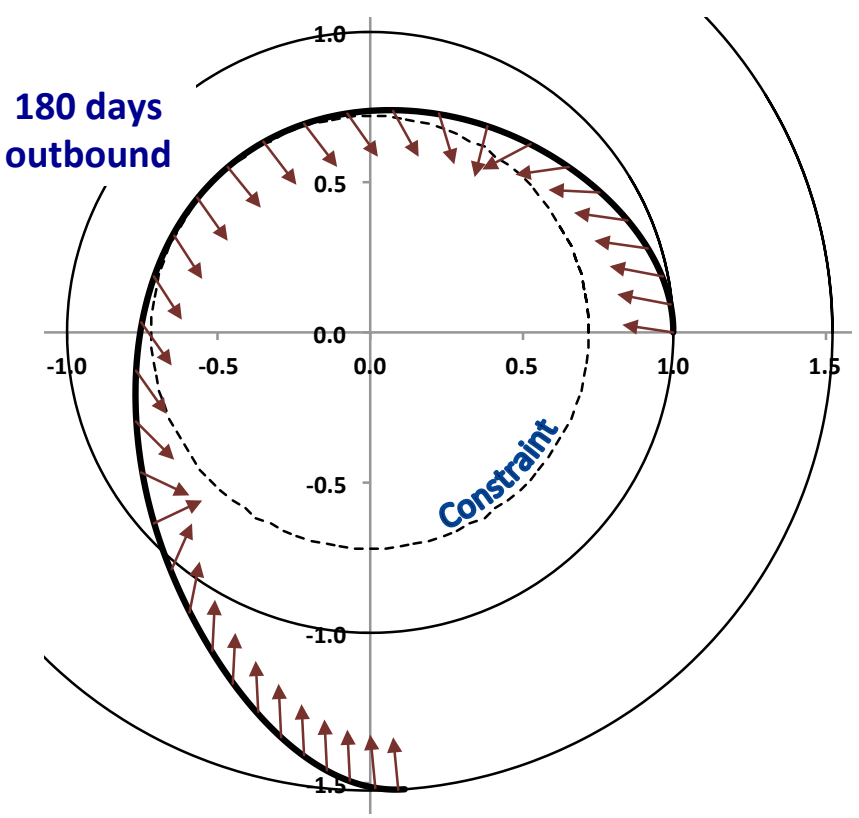

Figure 4 - Gravity Augmented Phasing

A distinctly different technique becomes apparent as departure phase angles are reduced from the phase-independent optimum (later departure dates). In these cases, orbital motion is resisted rather than augmented. The Q-Ship has sufficient performance to retard or actually reverse its heliocentric motion in order to allow Mars to advance relative to the vehicle's position in a maneuver we have termed "retro-phasing". Figure 5 depicts the trajectory associated with an initial phase angle of $-90^{\circ}$. This technique provides the minimum achievable flight times until departure phase angles decrease past $-135^{\circ}$ after which gravity-augmented phasing (with the vehicle travelling the other way around the sun!) is faster. 


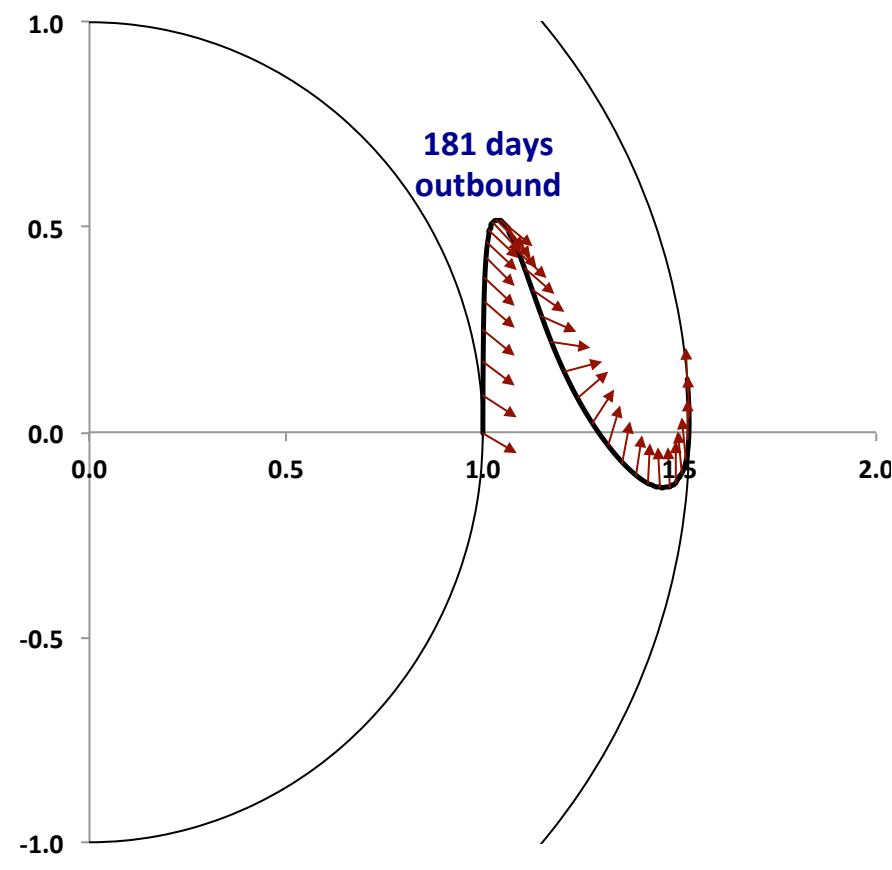

Figure 5 -"Retro-Phasing"

When we combine the techniques of gravity-augmentation and retro-phasing over the entire 26-month Earth-Mars synodic cycle $\left(360^{\circ}\right.$ of departure phase angles) we see that for the vehicle and mission parameters we have selected, the transit times vary from $2 \frac{1}{2}$ months at phase-optimum to 7 months at the "cross-over" of the phasing techniques (Figure 6). The implications should be stated clearly: there are no constraints on departure dates if Earth-Mars transit times of up to 7 months are acceptable! Also, since the QShip's constant thrust acceleration allows symmetry between outbound and return trajectories there are no constraints on Mars departure dates either, given the same transit time constraints.

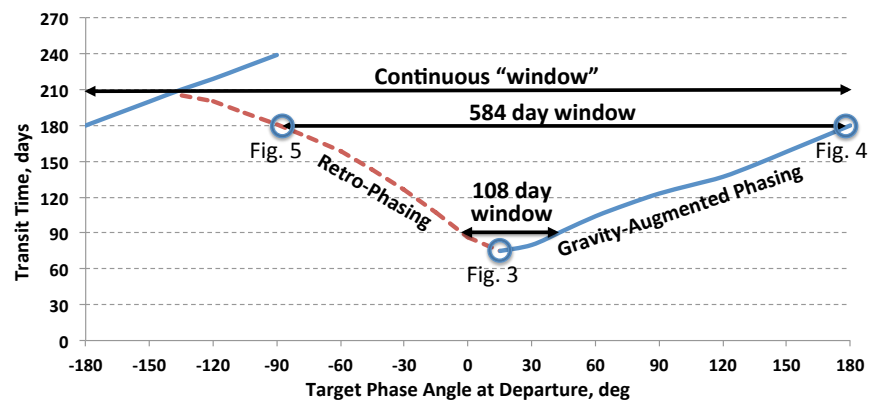

Figure 6 - Combined Phasing Techniques

\section{Crewed Q-Ship Mission}

While the fast Mars transits that Q-Thruster technology could enable would be revolutionary, the independence from the limitations of departure and arrival windows may ultimately be more so. This independence would allow missions to be conducted based on mission objectives and the limitations of crew health and safety rather than the laws of orbital mechanics. The concept of a "permanent" human presence at Mars with Q-Ships performing the crew rotation function - similar to International Space Station operations may be the best utilization of this capability. Using the results of the previous section, such a capability will be explored.

The function of the Q-Ships in this mission archetype would be to facilitate Mars crew exchanges. They would transport crews from low Earth orbit to low Mars orbit and return previous crew following their tours of duty. The sequence of events follows:

1) The Mars crew and supplies are transported to a QShip in low Earth orbit using an Earth-to-orbit infrastructure similar to that supporting the International Space Station.

2) When ready, the Q-Ship departs and transports the crew to low Mars orbit in a trip lasting from 3 to $71 / 2$ months depending on planetary phasing. These times include the planetocentric spirals.

3) The crew debarks to prepositioned infrastructure in low Mars orbit - an orbital habitat and/or planetary lander.

4) The previous crew, having completed their Mars tour of duty boards the Q-Ship. One month is allocated for this crew exchange.

5) Then when ready, the Q-Ship departs low Mars orbit and transports the returning crew to low Earth orbit in a trip lasting from 3 to $7 \frac{1}{2}$ months depending on planetary phasing. These times include the planetocentric spirals.

6) Earth-to-orbit transport is launched to the Q-Ship to return the Mars crew to Earth. This transport could also bring supplies and/or maintenance personnel to the Q-Ship.

7) Maintenance and resupply of the Q-Ship occur in low Earth orbit. When the Q-Ship is ready, the cycle repeats.

While this mission construct seems straightforward, it is not one that is often applied to Mars mission concepts, simply because traditional vehicle performance and dependence upon departure/return windows have not permitted reasonable length tours of duty. Of interest will be the tour of duty duration that can be supported with a single Q-Ship using the above concept of operations.

Figure 7 shows the same outbound transit time vs. departure phase angle curve as Figure 6, but superimposed is similar information for the return transit assuming a 30-day stay at Mars. The top curve is the superposition, which shows the total round-trip mission time as a function of departure phase angle. It is apparent that even with the "worst" departure phase angles a complete mission cycle can be 
completed in less than 390 days thereby allowing two round-trip missions per synodic cycle with a single vehicle. In addition, avoiding the adverse phase angles would allow crew tours of duty at Mars of around one year with 4-6 month transits each way - a seemingly reasonable scenario that would also provide several months between missions in low Earth orbit for vehicle turnaround. Figure 8 shows an example of the Q-Ship round-trip trajectory assuming a $180^{\circ}$ departure phase angle. If shorter Mars tours of duty were desired, a second Q-Ship could be inserted into the rotation.

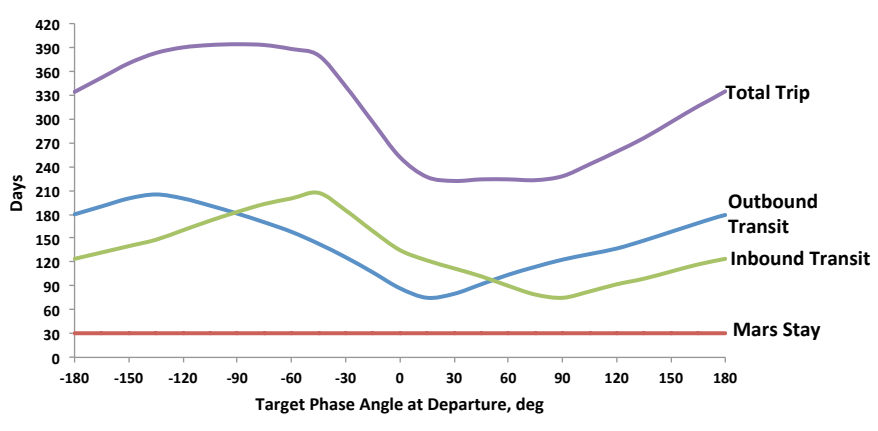

Figure 7 - Round-Trip Mission Times

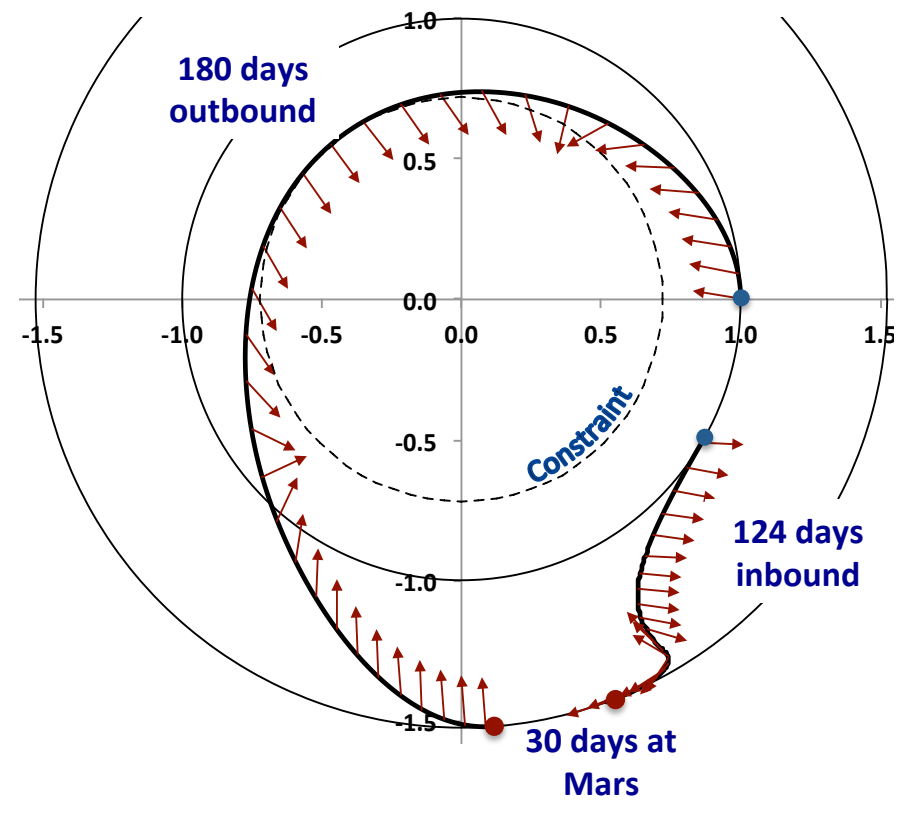

Figure 8 - Example Round-Trip Trajectory

\section{Q-Ship Cargo Capability}

There will be a need for significant cargo delivery capability to Mars to support the sort of crewed presence described above. This could include both crewed and cargo landers for surface mission support, orbital habitats for temporary crew billeting during rotations and lander support services, etc. A quick assessment was made of the cargo delivery capability of a Q-Ship having the same power and propulsion system parameters as those of the previous analyses, but with the crew module replaced with 100 tons of one- way cargo. This capability would encompass delivery of the largest surface landers envisioned in Ref DRA5 or a Skylabclass orbital habitat to low Mars orbit.

Figure 9 depicts the trajectory of such a cargo run. Using optimized outbound phasing, the 100-ton payload could be delivered in less than 5 months including the more extended spiral times at Earth and Mars. "Deadheading" back to Earth would require only 3 months. This is obviously not the most efficient way to use such a freighter, since it would be sitting idle in Earth orbit for 18 months waiting for the next phase-optimal departure. Analysis similar to the crewed vehicle should be performed, where flight times over a wide range of departure phasing are assessed to maximize the mission rate per vehicle. The payload per vehicle is likely an additional parameter of interest, since the probable true metric to be maximized is freight throughput per vehicle, such as tonnage per year.

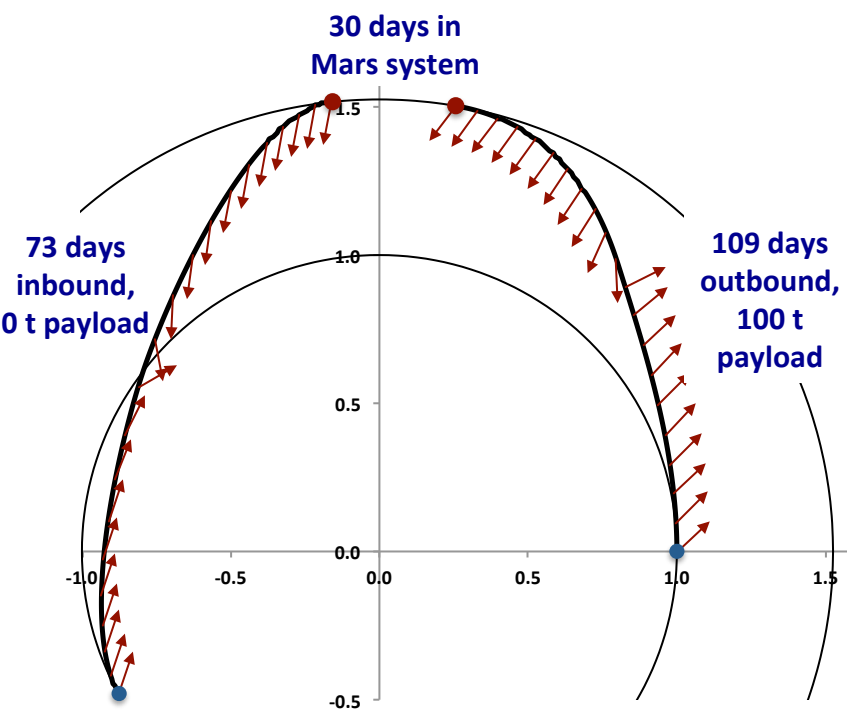

Figure 9 - Example 100 t Cargo Delivery Mission

\section{Extreme Performance Missions}

\section{Crewed Jupiter Missions}

The rapidly increasing scale of the solar system beyond Mars has traditionally been viewed as a barrier to human exploration. However, the combination of nuclear power and the performance characteristics of Q-Thrusters may change this. As evidenced by Figure 1, if milli-g levels of acceleration can be achieved, revolutionary transit times could be attained.

Similar to the Mars Q-Ship, we develop thrust and mass estimates for a crewed mission to Jupiter's moon Callisto. Callisto's orbit remains outside the most intense of Jupiter radiation belts, and therefore may represent a staging location for remote exploration of the other Galilean satellites. In any case it is likely that the mass of the habitation systems will be required to increase to accommodate the longer mission time, additional radiation protection, additional exploration equipment, etc. We chose 50 metric tons 
as the mass required for habitation systems, and to maintain thrust acceleration near the milli-g level the power to the thrusters was increased to 2 MWe. Q-Thuster specific thrust was maintained at $0.4 \mathrm{~N} / \mathrm{kWe}$ and power/propulsion system $\alpha$ at $20 \mathrm{~kg} / \mathrm{kWe}$. This results in $a_{\text {thrust }}=0.91 \mathrm{milli}-$ g's with a total vehicle mass of 90 metric tons.

Due to the expected flight times, the mission archetype chosen is more the traditional crewed round-trip rather than the crew exchange used for Mars. We somewhat arbitrarily selected six months duration at Callisto, although if this results in adverse phasing upon return to earth, the time could be adjusted. As can be seen in Figure 10, phenomenally rapid Jupiter transit times of around six months are achievable. While the outbound (and nearly the inbound) transit utilizes optimal phasing, this is not as constraining as it was with Mars missions since the Earth-Jupiter synodic period is only 13 months. The round trip can be accomplished within 20 months

\section{Crewed Saturn Mission}

Utilizing the same vehicle parameters as those of the Jupiter mission we constructed a crewed Saturn mission which is designed to spend six months at the Saturnian moon Titan and six months at Enceladus - both destinations of considerable scientific interest. Again, using nearly optimal phasing we see from Figure 11 that the transit to Saturn can be accomplished in only nine months. It is informative to note that at the turnaround point in the transit, our vehicle is travelling in excess of 100 kilometers per second - over five times the local solar system escape velocity.

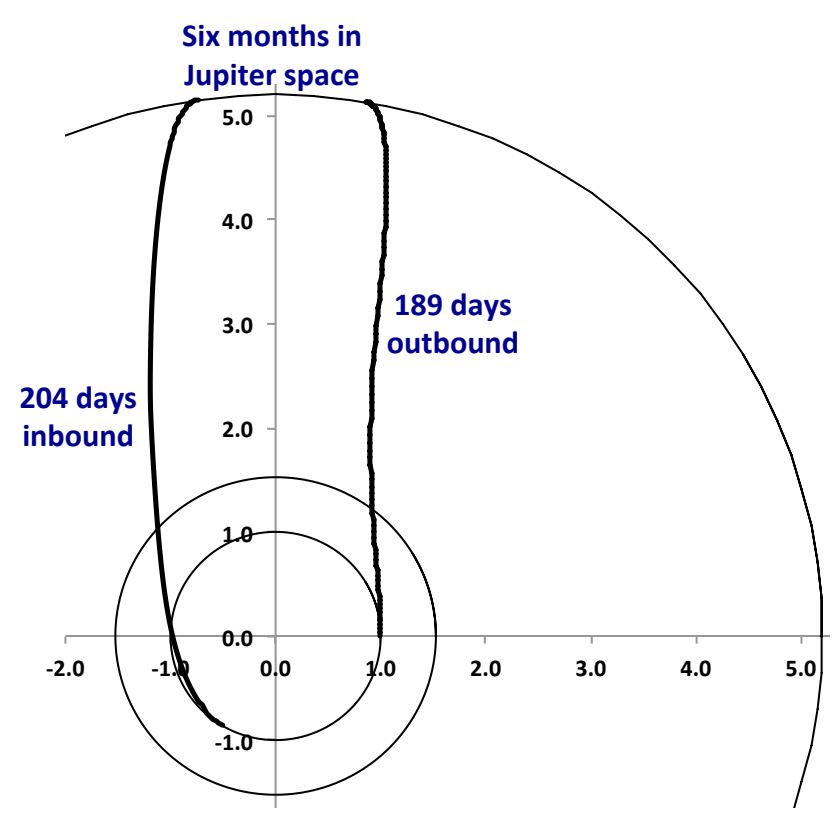

Figure 10 - Example Jupiter Mission

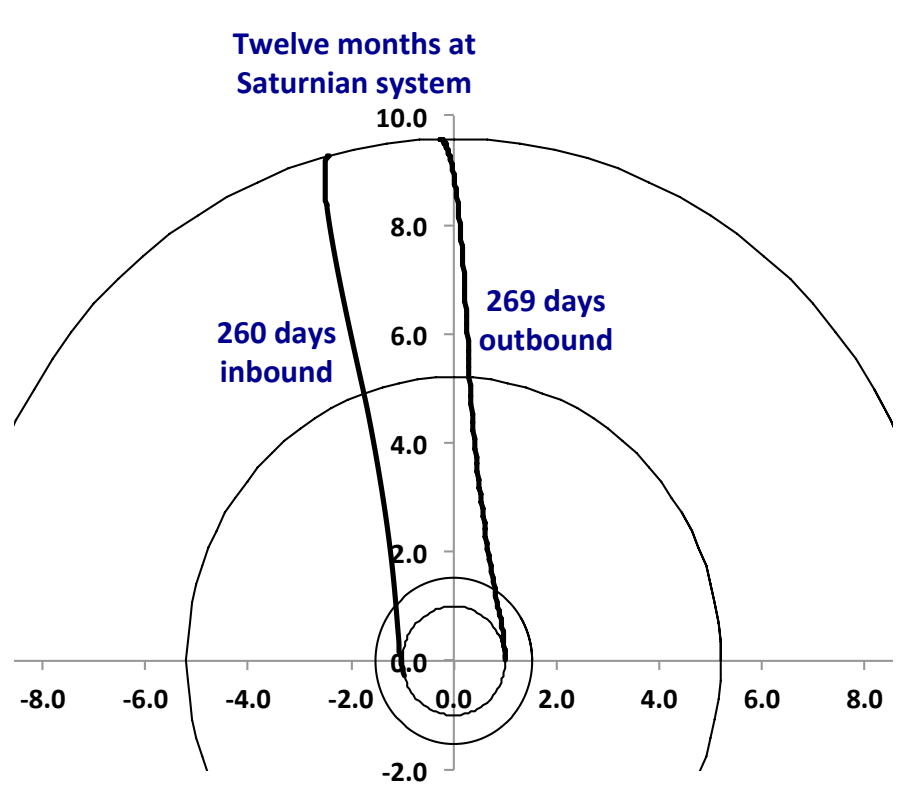

Figure 11 - Example Saturn Mission

\section{Interstellar Performance}

The velocities attained in only a few months for the Saturnian mission raise an interesting question as to those that could be reached for very long thrust periods. While no attempt is made here to design an interstellar probe, it is fairly straightforward to compute the velocities and transit time that could be achieved with milli-g levels of QThruster performance.

For most purposes, analysis of accelerated motion that may attain a significant fraction of lightspeed must use general relativity equations to predict spacecraft trajectories. However, for the restricted case of constant, rectilinear acceleration, special relativity relationships can be employed [15]. The spacecraft velocity $v$ can be expressed

$$
v=\frac{a_{\text {thrust }} \Delta \mathrm{t}}{\sqrt{1+\frac{a_{\text {thrust }}^{2} \Delta t^{2}}{c^{2}}}},
$$

where $a_{\text {thrust }}$ is the acceleration level experienced by the spacecraft in it's reference frame, $\Delta t$ is the transit duration as experienced in the rest frame and $c$ is lightspeed. The distance travelled, $z$ can be expressed

$$
z=\frac{c^{2}}{a_{\text {thrust }}}\left(\sqrt{1+\frac{a_{\text {thrust }}^{2} \Delta t^{2}}{c^{2}}}-1\right) .
$$

This can be solved for the time interval needed to travel a given distance under constant thrust:

$$
\Delta t=\sqrt{\frac{c^{2}}{a_{\text {thrust }}^{2}}\left[\left(\frac{z a_{\text {thrust }}}{c^{2}}+1\right)^{2}-1\right]} .
$$


For a one-way, non-decelerating trip to $\alpha$ Centauri, $\left(z_{\alpha C e n}=4.37\right.$ light years $)$ under a constant one milli-g acceleration, $\Delta t=92$ years with a velocity at the destination of $0.094 c$. If deceleration at the target system were desired, the trip would take 130 years, with a spacecraft velocity at the turnaround point of $0.067 c$. The Lorentz factors for the decelerating and non-decelerating missions are 1.0023 and 1.0045 , respectively, so relativity effects are minor.

While designing spacecraft systems for a $\sim 100$ year mission is certainly daunting, it should be pointed out that the U.S. Voyager 1 and 2 spacecraft continue to operate after onethird of that duration.

\section{SUMMARY}

While Quantum Thruster technology is currently at a relatively low level of technology readiness, it has been instructive to understand the potential implications to solar system transportation should postulated levels of specific thrust be attained. The combination of constant milli-g thrust, megawatt power levels and essentially zero propellant expenditure would provide revolutionary human transportation capabilities to and from the outer planets with typical round-trip mission durations of 10 months for Mars, 19 months for Jupiter and 30 months for Saturn. For Mars in particular, the resultant performance level would allow crewed missions independent of planetary phasing and highcapacity, frequent cargo delivery capabilities.

Further analyses should examine specific earth departure and return techniques. Unconstrained Q-Ship spiral trajectories could expose crewmembers to undesirable levels of radiation exposure due to the Van Allen radiation belts. It may be possible to use the Q-Thruster performance levels to design trajectories that avoid or minimize transits through the most problematic regions.

Since Mars missions traditionally display larger opportunity-dependent performance variations than missions to other planets due to Mars' orbital eccentricity and relative inclination it would be prudent to check the GRG/Excel ${ }^{\circledR}$ results against tools utilizing accurate planetary ephemerides, although it is expected that the postulated Q-Thruster performance would greatly attenuate these variations.

\section{REFERENCES}

[1] Millis, M. and E. Davis, ed., Frontiers of Propulsion Science, AIAA (2009).

[2] Brady, D. A., H. G. White, P. March, J. T. Lawrence and F. J. Davies, "Anomalous Thrust Production for an RF Test Device Measured on A Low-Thrust Torsion Pendulum." AIAA 2014-4029, 50 ${ }^{\text {th }}$ AIAA/ASME/SAE/ASEE Joint Propulsion Conference, July 28-30 2014.

[3] Zola, C. L. A Method of Approximating Propellant Requirements of Low-Thrust Trajectories. NASA TN-3400, April 1966.
[4] Gilland, J. H. "Mission and System Optimization of Nuclear Electric Propulsion Vehicles for Lunar and Mars Missions." Proceedings of the 22nd International Electric Propulsion Conference. IEPC 1991-038, 1991.

[5] Melbourne, W. G. Interplanetary Trajectories and Payload Capabilities of Advanced Propulsion Vehicles. NASA TR 32-68, March 1961.

[6] Lasdon, L. S., R. L. Fox, and M. W. Ratner. "Nonlinear optimization using the generalized reduced gradient method." Revue francaise d'automatique, d'infomatique et de recherce operationnelle. Recherche operationnelle. 8, no. 3 (1974): 73-103.

[7] Moyer, H. G., and G. Pinkham. "Several Optimization Techniques." In Computing Methods in Optimization Problems, by A. V. Balakrishnan and L. W. Neustadt (eds.), 65-105. New York: Academic Press, 1964.

[8] Bryson, A. E., and Y. C. Ho. Applied Optimal Control. Washington, D.C.: Hemisphere Publishing Corporation, 1975 .

[9] Ocampo, C. A., and J. Senent. "The Design and Development of Copernicus: A Comprehensive Trajectory Design and Optimization System." Proceedings of the International Astronautical Conference. International Astronautical Federation, 2006.

[10] George, J. A. Multi-Reactor System Configurations for Multimegawatt Nuclear Electric Propulsion. NASA TM105212, September 1991.

[11] Edelbaum T. N. "Propulsion Requirements for Controllable Satellites," ARS Journal, Vol. 31, August 1961, pp. 1079-1089.

\section{[12] DRA5}

[13] DRA5 appendix

[14] Ilin, A. V., L. D. Cassady, T. M. Glover, M. D. Carter and F. R. Chang-Diaz. "A Survey of Missions using VASIMR for Flexible Space Transportation," NASA JSC65825, April 2010.

[15] DeWitt, B. "Bryce DeWitt's Lectures on Gravitation", S. M. Christensen (ed.), Lecture Notes in Physics, 826, DOI: 10.1007/978-3-540-36911-0_2, CSpringer-Verlag Berlin Heidelberg 2011.

\section{BIOGRAPHY}

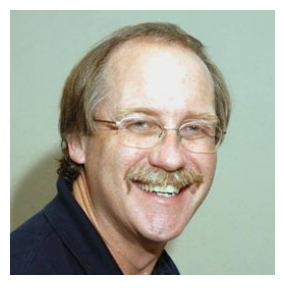

B. Kent Joosten holds Master of Science and Bachelor of Science degrees in Aerospace Engineering from Iowa State University. He is an independent consultant specializing in the support and development of advanced human space exploration architectures. This includes formulating strategic mission goals, performing preliminary mission concept development and identifying technology investment options. His clients have included both the U.S. Government and commercial 
aerospace companies. Prior to 2012 he was a senior systems engineer with the NASA Johnson Space Center specializing in human spaceflight mission design. He has been a participant in numerous advisory committees regarding the future of human spaceflight and was a flight controller for over 30 Space Shuttle missions.

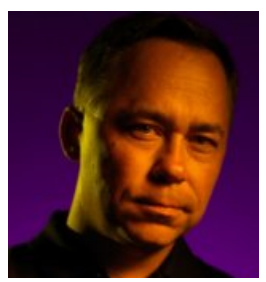

Dr. Harold G. Sonny White holds a Ph.D. in Physics from Rice University, a Master's of Science in Mechanical Engineering from Wichita State University, and a Bachelors of Science in Mechanical Engineering from University of South Alabama. He currently serves as the Advanced Propulsion Theme Lead for the NASA Engineering Directorate and is the JSC representative to the Nuclear Systems Working Group. In his role, Dr. White is serving to help the Agency incorporate high TRL advanced power and propulsion technologies into near and mid-term human exploration architectures. He is also pursuing theoretical and laboratory research on developing lower TRL advanced propulsion and power technologies in the advanced propulsion physics laboratory known as Eagleworks that is located at the Johnson Space Center. 


\section{APPENDiX A \\ Analysis of Conservation of Energy for Interplanetary Space Missions using Electric Propulsion}

The intent of this paper is a "what if" exploration of a possible emerging technology concept to some mission applications. This exercise helps to understand and articulate the capabilities that the performance metrics discussed here bring to the mission planner's toolbox. Although the performance metrics are introduced as attached to the concept of a Q-Thruster, any advanced propulsion system that has similar characteristics can also use this analysis effort to understand the mission benefits.

One of the issues to consider for a constant thrust system is the matter of conservation of energy. When will the spacecraft with its given power level reach a state where the integral of the input power over a given time frame increase the kinetic energy of the spacecraft such that the change in kinetic energy is greater than the integral of power? The thrust for the spacecraft will be the thrust-topower ratio times the power of the spacecraft:

$$
T=T_{S} P .
$$

The velocity for the spacecraft is a simple matter:

$$
v=\left(T_{s} P / m\right) t
$$

Where $t$ is the thrust duration. Using this velocity, the change in kinetic energy of the spacecraft is

$$
K . E .=T_{s}^{2} P^{2} t^{2} /(2 m) .
$$

The input energy from the power system for the same time period is simply $E=P t$. The point of interest is when these two energy changes are equal to one another:

$$
T_{S}^{2} P^{2} t^{2} /(2 m)=P t
$$

This condition occurs at a change in time of $t=$ $2 m /\left(T_{s}^{2} P\right)$ which equates to a $\Delta v$ of $2 / T_{s}$. When this situation occurs, in order to ensure that the input energy is equal to the change in kinetic energy, the thrust to power performance will have to decrease over time. This scenario has an analog in the terrestrial realm when considering a turbine aircraft flight profile. At takeoff, the turbine aircraft has a very high thrust to power (hundreds of $\mathrm{N} / \mathrm{kW}$ ), but at cruise altitude, the thrust to power performance is much lower $(1-10 \mathrm{~N} / \mathrm{kW})$. The following graph shows the curve with some highlighted data points for consideration.

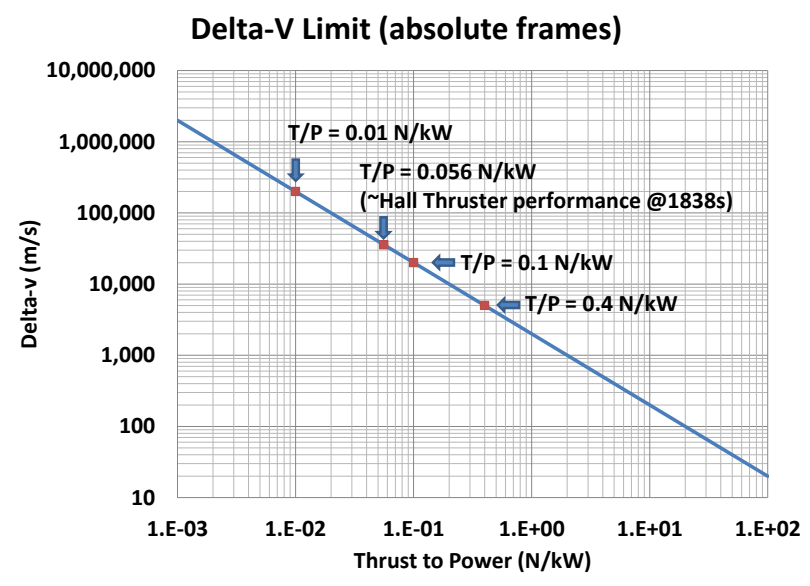

At this point, it is a useful exercise to explore this issue from a relativistic point of view, as it will uncover a paradox. For example, we know from study of the cosmic microwave background radiation that our Milky Way galaxy has a peculiar velocity (e.g. not from expansion of space, but a real velocity) of $\sim 371 \mathrm{~km} / \mathrm{s}$ as evidenced by the anisotropic measurements of the background radiation temperature. Consider what an inertial observer that is at rest relative to the background radiation would see when considering a spacecraft in our solar system that undergoes a $\Delta v$ of $1 \mathrm{~km} / \mathrm{s}$. The example spacecraft for our scenario will be a $10,000 \mathrm{~kg}$ spacecraft with a power system that provides $10 \mathrm{kWe}$ of power. The electric propulsion system for this example spacecraft will be modeled as Hall-thruster-like with thrust to power of $0.056 \mathrm{~N} / \mathrm{kWe}$ and a specific impulse of $1838 \mathrm{~s}$. This spacecraft will take 17,370,579 seconds or 201 days to change the velocity of the spacecraft by $1 \mathrm{~km} / \mathrm{s}$, and will consume $540 \mathrm{~kg}$ of propellant. The amount of energy provided by the power source over this time frame is 174 Gigajoules. The change in kinetic energy as measured by the inertial observer at rest relative to the background radiation is the initial kinetic energy $\left(\frac{1}{2} m_{i} v_{i}^{2}\right)$ minus the final kinetic energy $\left(\frac{1}{2} m_{f} v_{f}^{2}\right)$. The initial mass is 10,000 $\mathrm{kg}$, the final mass is $9,460 \mathrm{~kg}$. The initial velocity is 371 $\mathrm{km} / \mathrm{s}$, and the final velocity is $372 \mathrm{~km} / \mathrm{s}$, which assumes the spacecraft, had a radial trajectory aligned with the peculiar velocity vector. The change in kinetic energy is 33,649 Gigajoules, which is two orders of magnitude larger than the energy provided by the power system. Although the example mission is clearly not an exotic mission and can easily be achieved in practice, the point of this paragraph is to identify that the paradox can be created for any spacecraft using conventional propulsion as well as advanced propulsion. 


\section{APPENDIX B}

\section{Equations of Motion}

\section{Coordinate System}

As coordinate system for the translational motion, a flight path-oriented axis system is chosen. The x-axis is tangential to the trajectory, positive in the flight direction and inclined to the heliocentric radius vector normal plane by the flight path angle $\gamma$, positive for increasing radius. The azimuth angle $\chi$ designates the change in orientation of the non-radial velocity component relative to the initial time $t_{0}$, positive in the direction of the orbital angular momentum vector. The change in planar angle relative to $t_{0}$ is $\theta$, the change in out-of-plane angle is $\Lambda$.

Controls are the thrust directions $\varepsilon(t)$ (out of the instantaneous orbit plane) and $\sigma(t)$ (in the orbit plane).

\section{Equations of Motion}

The equations of motion for a thrusting vehicle in heliocentric space can then be expressed:

$$
\begin{gathered}
\dot{V}=-g \sin \gamma+\frac{T}{m} \cos \varepsilon \cos \sigma \\
\dot{\chi}=-\frac{V}{r} \cos \gamma \cos \chi \tan \Lambda+\frac{T}{m V} \frac{\sin \varepsilon}{\cos \gamma}, \\
\dot{\gamma}=\left(\frac{V}{r}-\frac{g}{V}\right) \cos \gamma+\frac{T}{m V} \cos \varepsilon \sin \sigma, \\
\dot{\theta}=\frac{V}{r} \frac{\cos \gamma}{\cos \Lambda} \cos \chi \\
\dot{\Lambda}=\frac{V}{r} \cos \gamma \sin \chi \\
\dot{r}=V \sin \gamma \\
\dot{m}=-c \\
g=\frac{\mu_{\odot}}{r^{2}}
\end{gathered}
$$

where $V$ is the magnitude of the vehicle velocity vector, $T$ is the thrust magnitude, $r$ is the radial distance from the vehicle to the sun, $m$ is the vehicle mass, $c$ is the propellant mass flow rate, $g$ is the magnitude of the sun's gravitational acceleration and $\mu_{\odot}$ is the sun's gravitational constant.
If motion is constrained to the original orbit plane, the equations simplify to:

$$
\begin{gathered}
\dot{V}=-g \sin \gamma+\frac{T}{m} \cos \sigma, \\
\dot{\gamma}=\left(\frac{V}{r}-\frac{g}{V}\right) \cos \gamma+\frac{T}{m V} \sin \sigma, \\
\dot{\theta}=\frac{V}{r} \cos \gamma, \\
\dot{r}=V \sin \gamma, \\
\dot{m}=-c, \\
g=\frac{\mu_{\odot}}{r^{2}} .
\end{gathered}
$$

\section{Canonical Units}

Normalizing the state variables can aid in stability of numerical integration and other calculations. The classic "canonical units" normalization has been utilized where, for heliocentric space:

The Distance Unit $\left(D U_{\odot}\right) \equiv 1 \mathrm{AU}$.

The Time Unit $\left(T U_{\odot}\right)$ is defined such that the orbital speed of an object in a $1 D U_{\odot}$ radius circular orbit is 1 $D U_{\odot} / T U_{\odot} . \quad$ That is, $1 T U_{\odot} \equiv \sqrt{1 D U_{\odot}{ }^{3} / \mu_{\odot}}$. So, in canonical units, $\mu_{\odot}=1 D U_{\odot}{ }^{3} / T U_{\odot}{ }^{2}$ and the orbital period of a $1 D U_{\odot}$ radius circular orbit is $2 \pi T U_{\odot}$.

Accelerations are scaled by $\mu_{\odot} / D U_{\odot}{ }^{2}$ or $1 D U_{\odot} / T U_{\odot}{ }^{2}$ which is the sun's gravitational acceleration at $1 D U_{\odot}$.

So, if $1 \mathrm{AU}=149,599,650 \mathrm{~km}$ and $\mu_{\odot}=132,715,440,000$ $\mathrm{km}^{3} / \mathrm{s}^{2}$, then

$1 D U_{\odot}=149,599,650 \mathrm{~km}$,

$1 T U_{\odot}=5,022,676 \mathrm{~s}$,

$1 D U_{\odot} / T U_{\odot}=29.78485 \mathrm{~km} / \mathrm{s}$,

$1 D U_{\odot} / T U_{\odot}{ }^{2}=5.93008 \times 10^{-6} \mathrm{~km} / \mathrm{s}^{2}$. 


\section{APPENDIX C}

\section{Test Problem}

\section{Test Problem}

To test the Excel ${ }^{\circledR}$-based optimal control solution method, we use one of the first numerically solved low-thrust trajectory optimization problems, described by Kopp and McGill [7] and subsequently solved in a slightly different form by Bryson and Ho [8]. In this problem, a spacecraft travels from earth's orbit around the sun to Mars' orbit using constant magnitude continuous low-thrust, $T$. The orbits of earth and Mars are considered to be circular and coplanar and the minimum time of flight is sought utilizing the thrust direction $\sigma(t)$ as the control.

Using the planar form of the state equations and heliocentric canonical units, the state variable initial conditions are:

$$
\begin{array}{r}
V\left(t_{0}\right)=1.0, \quad \gamma\left(t_{0}\right)=0.0, \quad \theta\left(t_{0}\right)=0.0, \\
r\left(t_{0}\right)=1.0 \text { and } \frac{m\left(t_{0}\right)}{T}=7.117
\end{array}
$$

with a normalized constant mass flow rate of $\frac{\dot{m}}{T}=0.533$.

Since we want to match the orbit of Mars, the desired state variable terminal constraints are:

$$
\begin{gathered}
\psi_{V}\left(t_{f}\right)=V\left(t_{f}\right)-\sqrt{\frac{1}{r\left(t_{f}\right)}}=0, \\
\psi_{\gamma}=\gamma\left(t_{f}\right)=0 \\
\psi_{r}=r\left(t_{f}\right)-1.525=0 .
\end{gathered}
$$

The performance index (to be minimized) is:

$$
\mathcal{J}=\phi\left[\bar{x}\left(t_{f}\right), t_{f}\right]+\int_{t_{0}}^{t_{f}} \mathcal{L}[\bar{x}(t), \bar{u}(t), t] d t
$$

using the calculus of variations terminology of Bryson and Ho [8], where $\bar{x}$ represents the vector of state variables, and $\bar{u}$ the vector of control variables. $\phi=0$ and $\mathcal{L}=1$ for this problem.

Moyer and Pinkham [7] employed a gradient technique along with penalty functions of the form:

$$
\phi_{P F}=\frac{1}{2} k_{V} \psi_{V}^{2}+\frac{1}{2} k_{\gamma} \psi_{\gamma}^{2}+\frac{1}{2} k_{r} \psi_{r}^{2},
$$

where the $k^{\prime} s$ are the penalty multipliers. Therefore their augmented penalty function is:

$$
\mathcal{J}=\phi_{P F}+\int_{t_{0}}^{t_{f}} d t
$$

In [7], the minimum flight time of 193 days was obtained.
Excel $^{\mathbb{R}}$ Solution - Solver

The state equations are implemented in Excel ${ }^{\circledR}$ through a fourth-order Runge-Kutta technique, with the equations themselves implemented in Visual Basic for Applications ${ }^{\circledR}$ (VBA) functions. The state equations can be generally expressed as:

$$
\frac{d \bar{x}}{d t}=f(\bar{x}, \bar{u}, t), \quad t_{0}<t<t_{f} .
$$

In general, the equations have a "free" final time, but by using a simple transformation they can be converted into fixed end-time to facilitate easier numerical evaluation. Let $t_{o}=0$ and define

$$
t=\eta \tau, \quad 0<\tau<1
$$

The quantity $\eta$ represents the final time and is treated as a control parameter and $\tau$ becomes the new independent variable. If, as in this problem, the state equations do not explicitly depend upon time, the transformed dynamics are simply

$$
\frac{d \bar{x}}{d \tau}=\eta f(\bar{x}, \bar{u})
$$

A constant one hundred-step integration interval was established which has proven adequate for the relatively smooth trajectory problems under consideration. A control function is therefore implemented as 101 discrete values at the integration nodes:

$$
u(\tau) \cong\left[u\left(\tau_{0}\right), u\left(\tau_{1}\right), u\left(\tau_{2}\right), \ldots u\left(\tau_{100}\right)\right]^{T}
$$

where $\tau_{0}=0$ and $\tau_{100}=1$. The initial state conditions and terminal state constraints are identical to the test problem in [7]. The performance index (to be minimized) is simply $\eta$, the final time.

Microsoft Excel Solver ${ }^{\circledR}$ uses the Generalized Reduced Gradient (GRG) Algorithm for optimizing nonlinear problems [6]. GRG determines directions of search by computing changes in the value of the performance index through finite differences of the control parameters, so user computation of the gradient of the Hamiltonian is unnecessary. The terminal state constraints are handled internally by means of "slack variables," so no penalty functions need to be developed.

To use Solver ${ }^{\circledR}$, the user sets up the spreadsheet so that the state variables and control function values are available at every integration node and the control parameter $\eta$, the values of the terminal state constraints and the value of the performance index are available in data cells.

When Solver ${ }^{\circledR}$ begins, it develops the finite difference information it needs to estimate search directions, and then begins the iterative process of minimizing the performance 
index while satisfying the terminal state constraints. These involve many solutions to the state equations, so they should be made as efficient and well-scaled as possible. If the user has set up plots of key state variables and the control, the time-histories can be observed changing during the optimization process.

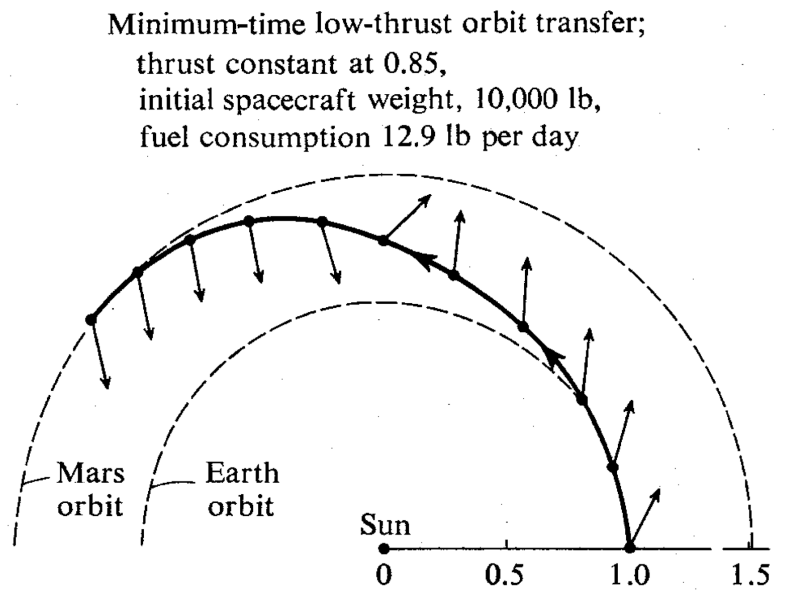

Trip time $=193$ days; thrust direction shown every 19.3 days Reference [8]
The test problem from [7] and [8] was set up and run using Solver/GRG ${ }^{\circledR}$. The results are shown below. The minimum flight time of 193 days was achieved, and the thrust direction time-history and trajectory is very similar to the [8] solution as shown in Figure 12.

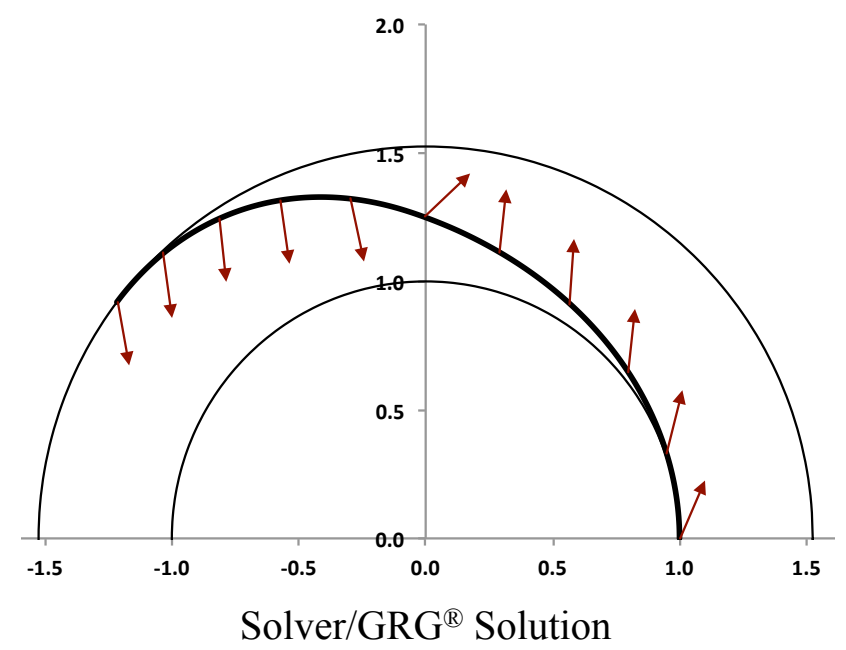

Figure 12 - Reference [8] Test Problem Comparison 